\title{
IMPLANTACIÓN DE LOS PORTAFOLIOS VIRTUALES DE APRENDIZAJE Y DE ENSEÑANZA EN ASIGNATURAS DE EDUCACIÓN SUPERIOR
}

\author{
José Alfredo Sarmiento Rodríguez \\ Especialista en Dirección de Empresas \\ Docente Investigador Universidad Santo Tomás, Seccional Bucaramanga \\ alfresar@gmail.com
}

\begin{abstract}
Resumen
Esta investigación diseñó y desarrolló para la plataforma Moodle, versiones del portafolio del estudiante, de la carpeta docente y de un instrumento de seguimiento que corresponde a herramientas importantes en el sistema de créditos académicos adoptado por la Universidad Santo Tomás, Bucaramanga. Dichos módulos de software fueron probados con estudiantes que realizaron durante dos semestres asignaturas del área de Derecho de la Facultad de Contaduría Pública, en ellas se aplicaron las mencionadas herramientas pedagógicas en papel y de manera virtual, con el $\mathrm{n}$ de hacer un comparativo y lograr observar la incidencia de los mismos en los procesos de metacognición de los estudiantes y del docente.
\end{abstract}

Palabras Clave: Portafolio virtual de aprendizaje, portafolio virtual de enseñanza, portafolio virtual, TIC y educación

\section{Summary}

This research, design and development for the platform Moodle versions of the student portfolio, teacher folder and a monitoring instrument that correspond to important tools in the academic credit system adopted by the University of Santo Tomas, Bucaramanga. These software modules were tested with students who performed for two semesters, subjects in the area of Law, School of Accounting. In these subjects the pedagogical tools already mentioned were applied paper based and virtually in order to make a comparison and observe their eect on the processes of students and teachers metacognition.

Key words: Virtual Learning Portfolio, virtual learning portfolio, virtual portfolio, ICT and education.

\section{Introducción}

El presente artículo de investigación congura la determinación del impacto en la metacognición de los estudiantes y el docente, al aplicar el portafolio de aprendizaje y de ense- ñanza en el método tradicional de aula y módulos desarrollados en la plataforma Moodle referidos al portafolio virtual de aprendizaje y al portafolio virtual de enseñanza, y la adición de un módulo de seguimiento, a estudiantes en asignaturas de Derecho de la Facultad de Contaduría Pública de la Universidad Santo Tomás.

La realización de este proyecto surgió a partir de tres aspectos. El primero: la Universidad Santo Tomás escogió a Moodle como LMS para apoyar el tiempo de trabajo independiente del estudiante; el segundo, que la

Pública como parte del desarrollo del sistema de créditos académicos adoptado por la Universidad según recomendación del Ministerio de Educación Nacional de Colombia y a través de sus docentes ha aplicado como didáctica el portafolio de enseñanza; y el tercero, que se llevó a cabo una consulta en la comunidad Moodle acerca de los desarrollos que se tenían al respecto, y al no encontrar uno que pudiera utilizarse con este $n$, llevó al autor a proponer a la Dirección de Investigaciones de la Universidad para una convocatoria interna y patrocinada por la Institución, una investigación cuyo proyecto se denominó: "Adaptación de la herramienta tecnológica de apoyo virtual Moodle al sistema de créditos en las asignaturas de Derecho Comercial y Derecho Laboral, de la Facultad de Contaduría Pública de la Universidad Santo Tomás de Bucaramanga."

En el aspecto metodológico la investigación realizada fue de carácter inductivo, cualitativo

LMS: Learning Management System, 
y cuasi experimental. De carácter inductivo, porque tuvo que ver con el seguimiento de una metodología mediante la construcción de etapas de observación de los hechos, y registro de los mismos, se analizó la información producida, se generaron deniciones claras de esos hechos, se crearon categorías de análisis y se aunaron en las conclusiones del proyecto. Las observaciones se realizaron sobre la experiencia tradicional enriquecida con el estudio de caso a partir de instrumentos de registro como el mismo portafolio, la carpeta docente, apuntes de la docente y entrevistas. De la misma manera se llevó la experiencia con el acompañamiento tecnológico que deja rastros observables, se crearon categorías de análisis de las mismas en el portafolio, carpeta y entrevistas, y se generaron de allí, algunas conclusiones independientes que al unirse nalmente aportaron a las conclusiones del proyecto.

La investigación cualitativa evita la cuancación. Por tanto, se hicieron registros narrativos de los fenómenos que se estudiaron mediante técnicas como la observación participante y las entrevistas no estructuradas en los respectivos contextos estructurales y situacionales. Con esta investigación se intentó identicar la naturaleza profunda de algunas realidades, su sistema de relaciones y su estructura dinámica, esto se pudo realizar mediante la observación por parte de los docentes investigadores, los asesores y el par invitado, además, con la aplicación de entrevistas a los grupos.

Se hace referencia a Cuasi experimental, ya que esta metodología se ha aplicó porque en el diseño de la investigación se carece de un control experimental absoluto de todas las variables relevantes, debido al carácter aleatorio de la selección de los sujetos, o en la asignación de los mismos para los grupos experimental y de control, que para el caso, hacen referencia a los grupos de Derecho Laboral y Derecho Comercial II, los cuales han sido asignados por la Universidad.
En cuanto al desarrollo de la investigación se realizó con los diseños del Portafolio de enseñanza y de aprendizaje. Con respecto al de aprendizaje, se plantearon tres requerimientos para ser tramitados: el primero especica el perl personal de cada estudiante y la posibilidad de consultar el de sus compañeros; el segundo indica que es necesario que el estudiante realice las entradas que considera necesarias alrededor de los Núcleos Temáticos que maneja la asignatura, también se consideró necesario que el estudiante tenga un espacio propio en el cual pueda adjuntar los archivos (Formatos Web estándar) que considere de interés alrededor de las temáticas que abarcan la asignatura. Como tercero, que cada vez que se realice una reexión en la bitácora y por núcleo temático, el estudiante debe responder cuestiones claves en torno al proceso de metacognición en donde se determinó que eran necesarios tres de estos espacios con los siguientes interrogantes: ¿Cómo planeo estudiar o realizar las actividades? ¿A qué me comprometo para mejorar mi aprendizaje? ¿Qué aciertos o desaciertos tuve al estudiar o realizar la actividad?

El anterior portafolio fue diligenciado por los estudiantes al nalizar el desarrollo de los ejes temáticos de Contratos para el caso de Derecho Comercial y de Derecho Colectivo, para el caso de la asignatura Derecho Laboral.

En el Portafolio de Enseñanza, el profesor tramitó tres requerimientos especícos, el primer requerimiento tiene que ver con su currículo en el cual se plasma su experiencia profesional y docente que será consultada por los estudiantes. En el segundo, los archivos que considere necesarios para consulta de sus estudiantes como base teórica y como tercer requerimiento, para consignar su proceso de enseñanza en el cual se contemplaron como necesarios tres espacios con los siguientes interrogantes: ¿Qué aciertos o desaciertos tuve al desarrollar el núcleo temático? ¿Cómo planeo enseñar el núcleo temático? ¿Qué re- 
quiero para favorecer el aprendizaje en los siguientes núcleos temáticos? Se consideró que el Profesor puede responder estas preguntas cada vez que lo considere necesario, alrededor del desarrollo de los Núcleos Temáticos que contenga la asignatura que maneje.

Con respecto a las conclusiones generales, el proyecto logró determinar el impacto de la incorporación de algunas herramientas de Moodle en procesos de metacognición de estudiantes de la Facultad de Contaduría Pública de la USTA en las asignaturas de Derecho Comercial II y Derecho Laboral, por cuanto los estudiantes, al comparar sobre experiencias anteriores, y con diversos docentes, perciben una gran diferencia en el autoanálisis de su proceso de aprendizaje que hasta ahora hacen y deja de ser exclusivo sólo entre los estudiantes disciplinados y se maniesta en todo el grupo, para el docente también es nuevo el aporte en la reexión de sus compromisos que quedan evidenciados y mueven a su acción, no quedan en la intención como sucede con las metodologías tradicionales en las que el docente es consciente de cambios pero difícilmente los realiza, la reexión acerca de los aciertos que el estudiantado encuentra en su propio desempeño toca distintos aspectos entre los que se encuentra la posibilidad de aanzar el desarrollo cognitivo, por cuanto se permitió el entendimiento completo del tema visto.

\section{Metodología}

La investigación realizada fue de carácter inductivo, cualitativo y cuasi experimental. En ella se aplicaron técnicas como las de observación y entrevista, e instrumentos de portafolio y carpeta, rastros de la plataforma Moodle, pretest y postest, y evaluaciones. La muestra de la investigación se conformó por un grupo control (Focal) y uno experimental en cada experiencia (tradicional y con tecnología). Las muestras fueron aleatorias entre 6 y 12 estudiantes para aplicación de entrevistas y análisis de datos.
En la investigación se realizó una observación en el tiempo de cuatro semanas, tiempo de duración de los módulos diseñados tanto para la experiencia tradicional como la experiencia en la plataforma, estas experiencias se dieron normalmente en el desarrollo del núcleo temático de cada asignatura como en el registro de las experiencias en el portafolio del estudiante y en la carpeta docente.

En cuanto al grupo focal y experimental y la estadística de muestras, se seleccionaron grupos entre 6 y 12 participantes, su selección fue de manera aleatoria aplicada en Excel una semilla de aleatorización a la lista de cada grupo e invitándolos a la participación. Además de esto, en el grupo experimental de la segunda experiencia participó un estudiante que tuvo la oportunidad de vivir la vivencia tomando las asignaturas al contrario de lo propuesto en el plan de estudios, es decir, primero cursó Derecho Comercial II y luego Derecho Laboral.

Con respecto a los instrumentos de recolección de la información, la entrevista se realizó no estructurada y focalizada, con una guía de temas, en este caso los investigadores se apoyaron en la pedagogía y la teoría. La entrevista es una técnica ecaz para obtener datos relevantes y signicativos desde el punto de vista de las ciencias sociales, la información que el entrevistador obtiene a través de la entrevista es muy superior a la adquirida cuando se limita a la lectura de respuesta escrita, su condición es oral y verbal, mediante la entrevista se pueden captar los gestos, los tonos de voz, los énfasis y demás, que aportan una importante información sobre el tema y las personas entrevistadas.

Se realizaron varias fases en la investigación, la primera de ellas tuvo que ver con el análisis de los núcleos temáticos de las asignaturas que se aplicarían en la investigación; en la segunda, se rediseñaron los materiales de apoyo de cada asignatura, basados en la didáctica de caso, lecturas de apoyo, guías de tiempo independiente, evaluaciones y demás componentes 
necesarios para la didáctica mencionada; en la tercera, se aplicó esta reestructuración en la primera experiencia que hemos llamado tradicional; en la cuarta, se realizaron los diseños, desarrollos y pruebas en Moodle tanto de los núcleos como de las herramientas de seguimiento, de portafolio del estudiante y de carpeta docente; en la quinta, se hizo la implementación de los núcleos y herramientas en Moodle; en la sexta, se puso en marcha la segunda experiencia apoyada en tecnología; y por último, se realizaron los análisis de la información generada y construcción de documentos finales de la investigación.

Con respecto a los aspectos técnicos y para el funcionamiento de las aplicaciones desarrolladas, se trabajó sobre la plataforma Moodle versión 1.8, pero los módulos funcionan sobre ésta o superior (Implica tener la versión 2.0 o superior del Servidor Apache, versión 5.0 o superior de lenguaje PHP), se trabajó sobre un sitio Moodle basado en el manejador MySQL Versión 4.2 (podría ser superior), por parte del cliente, y se usó el Navegador Web Internet Explorer 5.0 (también admite superior) ó Mozilla Firefox 2.0 o superior para poder manejar el editor de texto dentro de las aplicaciones.

El equipo de trabajo se conformó con un docente con experiencia en plataforma Moodle y académica, con asignación de tiempo completo como director del proyecto, y fue complementado por una docente y abogada del área de derecho de la facultad quien aplicó en sus materias el conocimiento generado, se adicionó a ella una pedagoga y un ingeniero de sistemas, quienes como grupo interdisciplinario enriquecieron la experiencia.

\section{Denición de poblaciones y muestras}

Los grupos de las asignaturas correspondientes a la población de la investigación, estuvieron conformados así:
En la primera experiencia, la asignatura de Derecho Laboral tuvo 25 estudiantes asistentes y la materia de Derecho Comercial 22 estudiantes asistentes, en la segunda experiencia, la asignatura de Derecho Laboral tuvo 30 estudiantes asistentes y la de Derecho Comercial 25 estudiantes asistentes.

\section{Resultados}

Los resultados obtenidos con respecto a los módulos desarrollados para Moodle como el portafolio del estudiante y la carpeta docente se plasman en los párrafos que siguen, en ellos se destacan: Imágenes de las aplicaciones en funcionamiento, imágenes del diseño de las bitácoras, sus funcionalidades, los formatos de evaluación aplicados a su uso y los resultados obtenidos por la aplicación de las herramientas de recolección de información usadas en las diferentes etapas del proceso.

\section{Portafolio del Estudiante}

Se muestra en la siguiente imagen el diseño de la bitácora que debe llevar el estudiante:

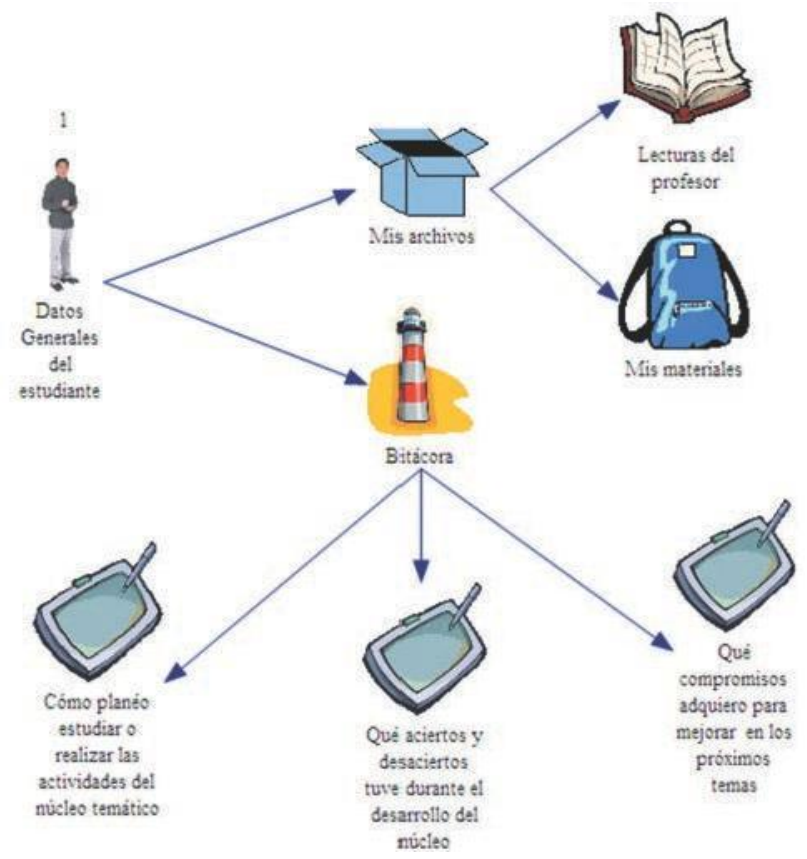

La funcionalidad de la herramienta llamada portafolio de aprendizaje o portafolio del estudiante, está dividida en los dos roles de usuario: 


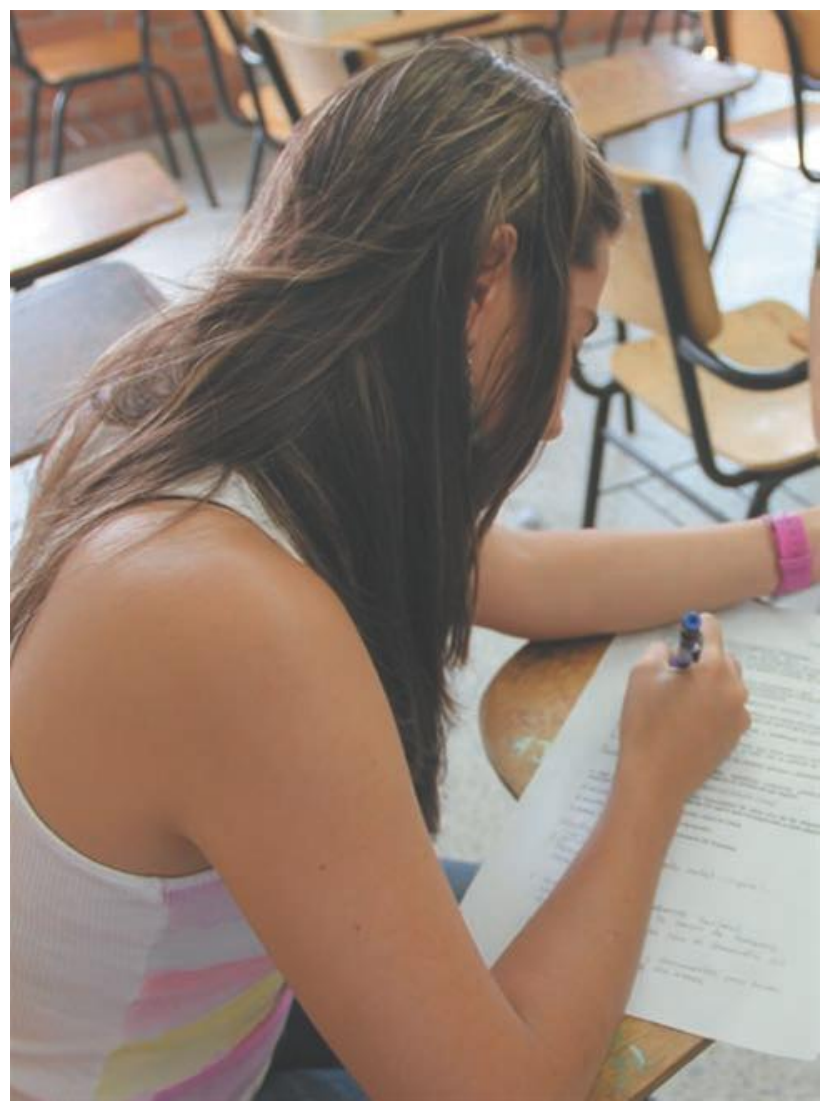

Rol de estudiante: es quien va a desarrollar el portafolio. Las acciones que permite la aplicación para este tipo de usuario son:

- Un espacio donde el estudiante pueda subir archivos y crear directorio dentro del servidor

- Accesoparapoderdescargarlosarchivos que deje a disposición el profesor

- UnespaciodondepuedaescogerunNúcleo Temático, editar los espacios de reflexión y llenar la bitácora

- Unespaciodondepuedaverlosresultados de la rejilla de evaluación

- Unespaciodondepuedaautoevaluarsu desempeño en el Portafolio

- Unespaciodondepuedaeditarsusdatos generales
Rol de Profesor: es quien va a revisar y controlar el desarrollo del portafolio en el curso. Va a desempeñar una serie de tareas:

- Una interfaz en la que pueda ver la lista de estudiantes que conforman el curso, con vínculos a las entradas que han realizado

- U n espacio para evaluar el portafolio a partir de una rejilla de valoración

- Unainterfazpararetroalimentarlasentradas realizadas por los estudiantes

- Unespaciodondepuedahacercopiasde seguridad del portafolio

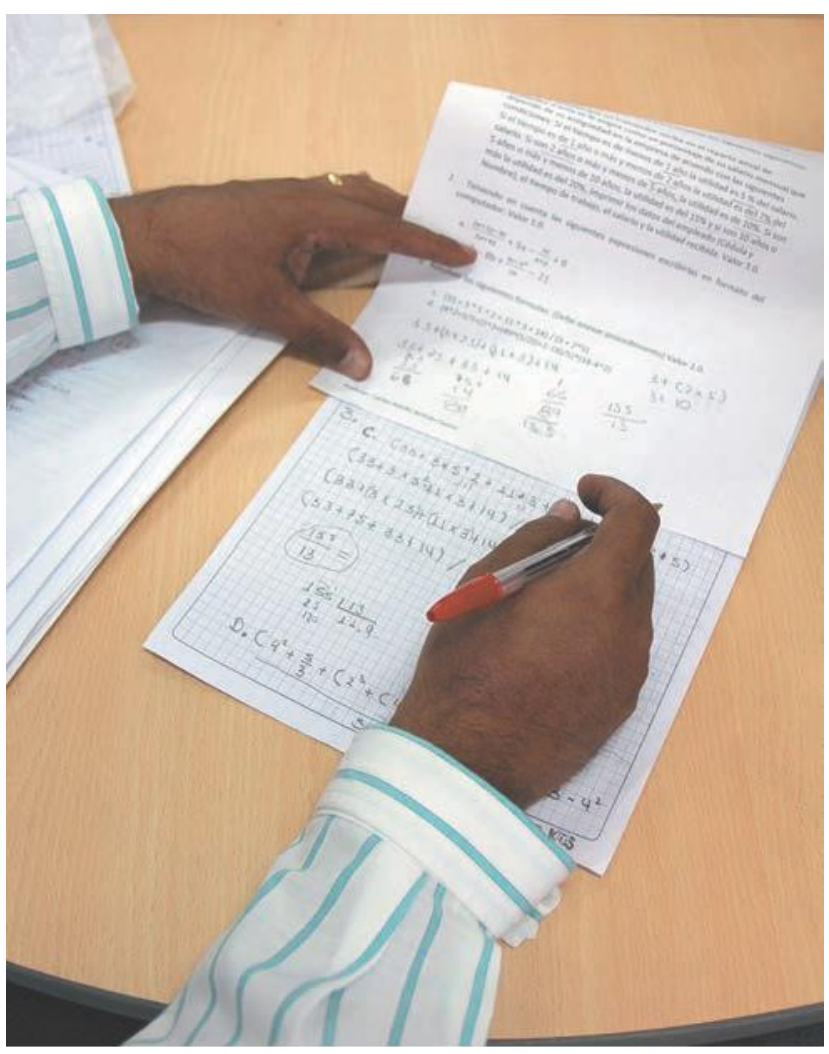

Esta imagen tiene que ver con el rol del estudiante, observando su portafolio de aprendizaje.

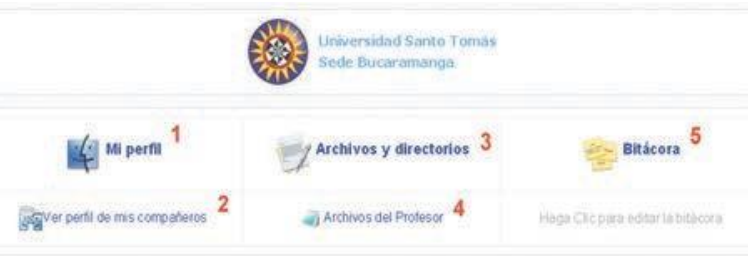


En la anterior imagen se encuentran tres requerimientos para ser tramitados: el primero especifica el perfil personal de cada estudiante y la posibilidad de consultar el de sus compañeros, el segundo indica que es necesario que el estudiante realice las entradas que considera necesarias alrededor de los Núcleos Temáticos que maneje la asignatura, también se consideró necesario que el estudiante tenga un espacio propio en el cual pueda adjuntar los archivos (Formatos Web estándar) que considere de interés alrededor de las temáticas que abarcan la asignatura, y como tercero, que cada vez que se realice una reflexión en la bitácora y por núcleo temático, el estudiante se debe responder cuestiones claves en torno al proceso de metacognición en donde se determinó que eran necesarios tres de estos espacios con los siguientes interrogantes:

¿Cómo planeo estudiar o realizar las actividades?

¿A qué me comprometo para mejorar mi aprendizaje?

¿Qué aciertos o desaciertos tuve al estudiar o realizar la actividad?

Los cuales se encuentran plasmados en la siguiente imagen:

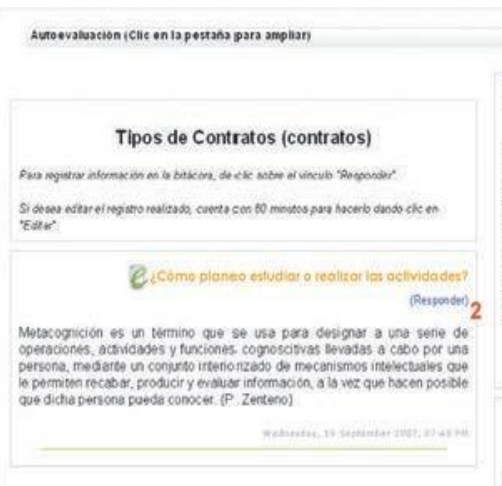

Se creó un formato de evaluación que permitiera valorar el uso del portafolio por parte del estudiante que lo aplica, de sus compañeros y del docente que imparte la asignatura,

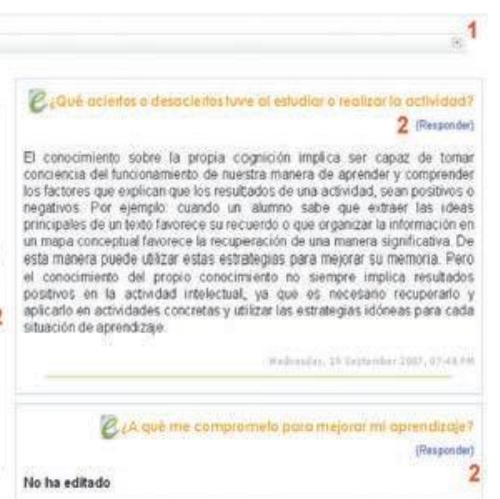

dicho formato se puede aplicar en heteroevaluación, autoevaluación y coevaluación, y se muestra a continuación:

Escala de valoración del Portafolio del Estudiante

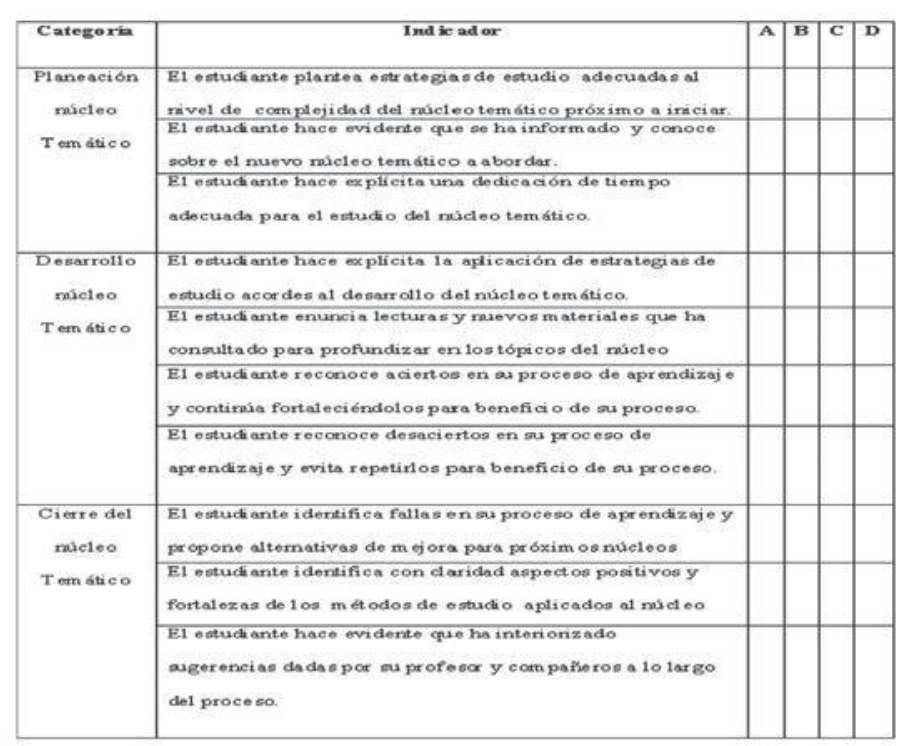


Y la medición del cumplimiento se realiza con los siguientes indicadores:

\begin{tabular}{|c|l|}
\hline $\begin{array}{c}\text { NIVEL DE } \\
\text { CUMPLIMIENTO }\end{array}$ & DESCRIPCIÓN \\
\hline A & El estudiante evidencia con sufciencia los indicadores de metacognición y supera los alcances formulados \\
\hline B & $\begin{array}{l}\text { El estudiante presenta alcances signifcativos en el cumplimiento de los indicadores de metacognición } \\
\text { formulados }\end{array}$ \\
\hline C & El estudiante muestra un bajo nivel de alcance de los indicadores de metacognición \\
\hline D & El estudiante no evidencia los indicadores de metacognición formulados \\
\hline
\end{tabular}

\section{Aciertos del estudiante}

La reexión acerca de los aciertos que el estudiantado encuentra en su propio desempeño toca distintos aspectos. Uno de ellos cita como acierto la posibilidad de aanzar el desarrollo cognitivo, por cuanto se permitió el entendimiento completo del tema visto, lo que rearma de la metodología utilizada, entre los aspectos principales que cita Brown (1983):

"La meta-cognición se reere al conocimiento de uno mismo y al control del dominio cognitivo (...). Aunque el conocimiento y la regulación del conocimiento están incestuosamente relacionados, esas dos formas de actividad tienen raíces bastante diferentes y los problemas que los acompañan son distintos. La tensión generada por el uso del mismo término, metacognición, para los dos tipos de conducta está bien ilustrada por el hecho de que incluso los más destacados ponentes en este campo tienden a responder a las cuestiones sobre la naturaleza de la metacognición con un "depende". ¿Se desarrolla tardíamente la meta-cognición? depende del tipo de conocimiento o proceso al que uno se reera. ¿Es consciente la metacognición? Depende (...) " (Brown, Bransford, Ferrara y Campione, 1983: 106-107).

De esta manera se distingue dos posibles áreas: el saber sobre el conocimiento y la regulación del conocimiento, se establecen diferencias entre un conocimiento factual y declarativo, y un conocimiento procedimental.
Y por último, se denota en los comentarios de los estudiantes, reexiones personales más profundas sobre:

- Lamateriaaportóalentendimientodequela carrera elegida fue la mejor opción de vida.

- Elesmeroporaprender,laactitudproactiva del estudiante y el esfuerzo en el logro estructurado de objetivos genera resultados positivos.

\section{Carpeta Docente}

Con respecto a la funcionalidad de la herramienta llamada Portafolio de enseñanza o carpeta docente permite unos espacios para el profesor:

- Para que pueda subir archivos dentro de carpetas previamente creadas para cada Núcleo Temático. Estos recursos quedan disponibles para ser revisados por el Estudiante.

- PuedaescogerunNúcleoTemáticoyeditar los espacios de reexión sobre el desarrollo de las clases.

- Puedacompletarlaautoevaluaciónsobreel manejo de la Carpeta por cada Núcleo Temático.

- Puedaeditarsucurrículoacadémico.

Esta imagen tiene que ver con el rol del profesor, que observa su portafolio de enseñanza. 


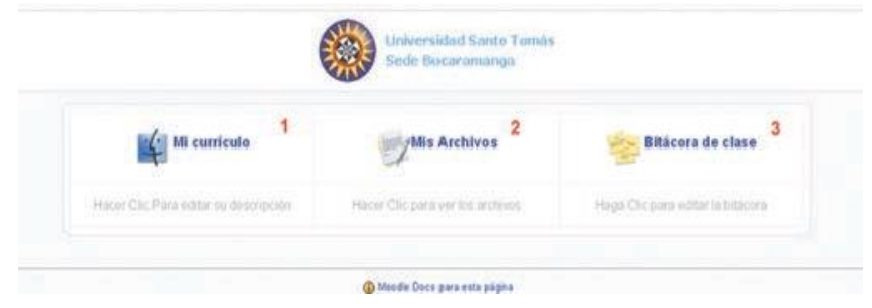

De similar forma que la aplicación creada para el Estudiante, el Profesor tramitará tres requerimientos especícos, el primero tiene que ver con su currículo en el cual se plasma su experiencia profesional y docente que será consultada por los estudiantes, en el segundo los archivos que considere necesarios para consulta de sus estudiantes como base teórica y como tercero, para consignar su proceso de enseñanza en el cual se contemplaron como necesarios tres espacios con los siguientes interrogantes: ¿Qué aciertos o desaciertos tuve al desarrollar el núcleo temático? ¿Cómo planeo enseñar el núcleo temático? ¿A qué requiero para favorecer el aprendizaje en los siguientes núcleos temáticos? Se consideró que el Profesor puede responder estas preguntas cada vez que lo considere necesario, alrede- dor del desarrollo de los Núcleos Temáticos que contenga la asignatura que maneje.

En el Portafolio del Estudiante se mencionó que existía un espacio donde los estudiantes pueden ver algunos archivos seleccionados por el profesor como complemento para el desarrollo la asignatura. Es aquí donde la Carpeta Docente debe interactuar con el Portafolio ya que el profesor enlaza estos archivos desde su aplicación.

También se determinó diseñar una rejilla para que el profesor se autoevalúe de su proceso de enseñanza alrededor de cada Núcleo Temático que abarque la asignatura, se muestra en la siguiente imagen:

Escala de valoración de la Carpeta Docente

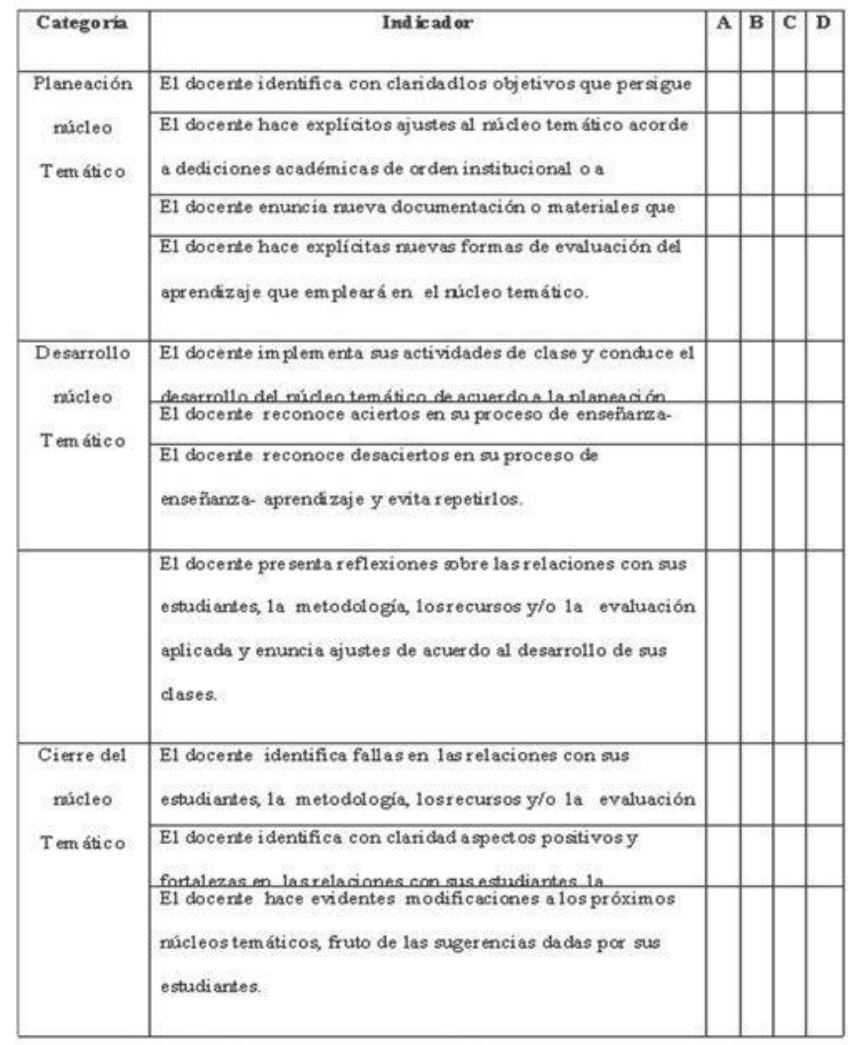


Y la medición del cumplimiento se realiza con los siguientes indicadores:

\begin{tabular}{|c|l|}
\hline $\begin{array}{c}\text { NIVEL DE } \\
\text { CUMPLIMIENTO }\end{array}$ & \multicolumn{1}{c|}{ DESCRIPCIÓN } \\
\hline A & El docente evidencia con sufciencia los indicadores de metacognición y supera los alcances formulados \\
\hline B & $\begin{array}{l}\text { El docente presenta alcances signifcativos en el cumplimiento de los indicadores de metacognición for- } \\
\text { mulados }\end{array}$ \\
\hline C & El docente muestra un bajo nivel de alcance de los indicadores de metacognición \\
\hline D & El docente no evidencia los indicadores de metacognición formulados \\
\hline
\end{tabular}

\section{Módulo de Seguimiento}

El módulo de seguimiento está valorado por la docente en análisis previos de portafolio y carpeta, el sentido principal y su uso está dado por el interés del docente al poder agrupar la información de la plataforma tomando la información pertinente por cuanto Moodle archiva en sus bases de datos la información de las transacciones realizadas, pero al igual que los sistemas de toma de decisiones empresariales, este módulo trasciende la sola recolección de información básica de la plataforma y agrupa la información en indicadores valiosos para el docente, por alumno y actividad y le permite comparar su actividad en el grupo, esto hace que el docente motive al estudiante que no participa y al que participa, llame la atención al que no lo hace y el grupo tenga esa sensación de que el docente, aún apoyado por una plataforma de apoyo tecnológico está relativamente presente, entienda que todas las fechas están registradas y que se pueden imprimir informes de actividades de diversas maneras, esto facilita la actividad de seguimien- to del docente al tiempo independiente que se valora tanto en el sistema de créditos de la Universidad en este momento.

La aplicación de los desarrollos de los módulos en Moodle, la intervención pedagógica para aplicación en plataforma de las asignaturas, la actitud de la docente y estudiantes durante la investigación, así como la colaboración del equipo de personas implicados en la investigación generaron las conclusiones que mencionamos a continuación.

El primero de ellos es que el proyecto logró determinar el impacto de la incorporación de algunas herramientas de Moodle en procesos

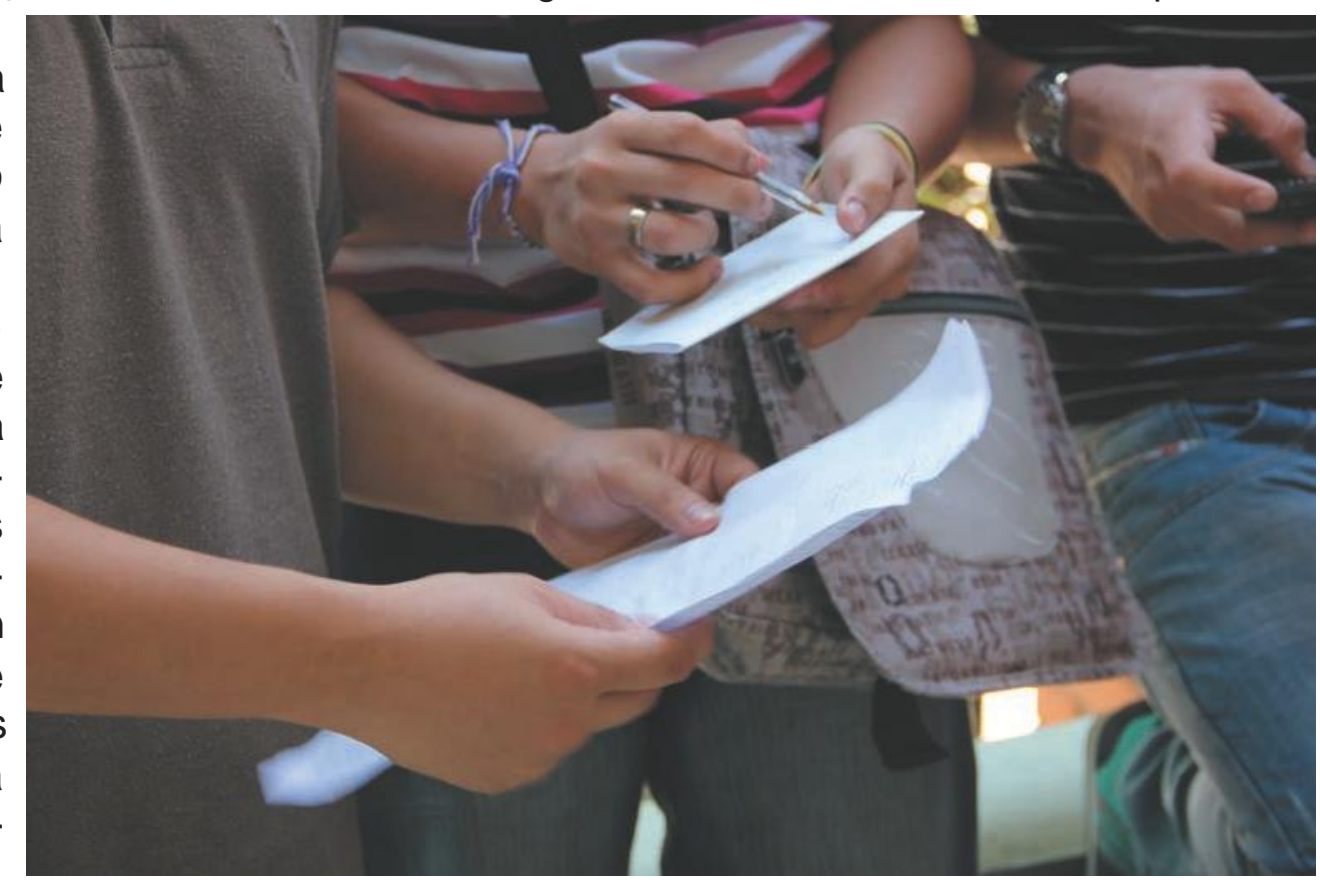




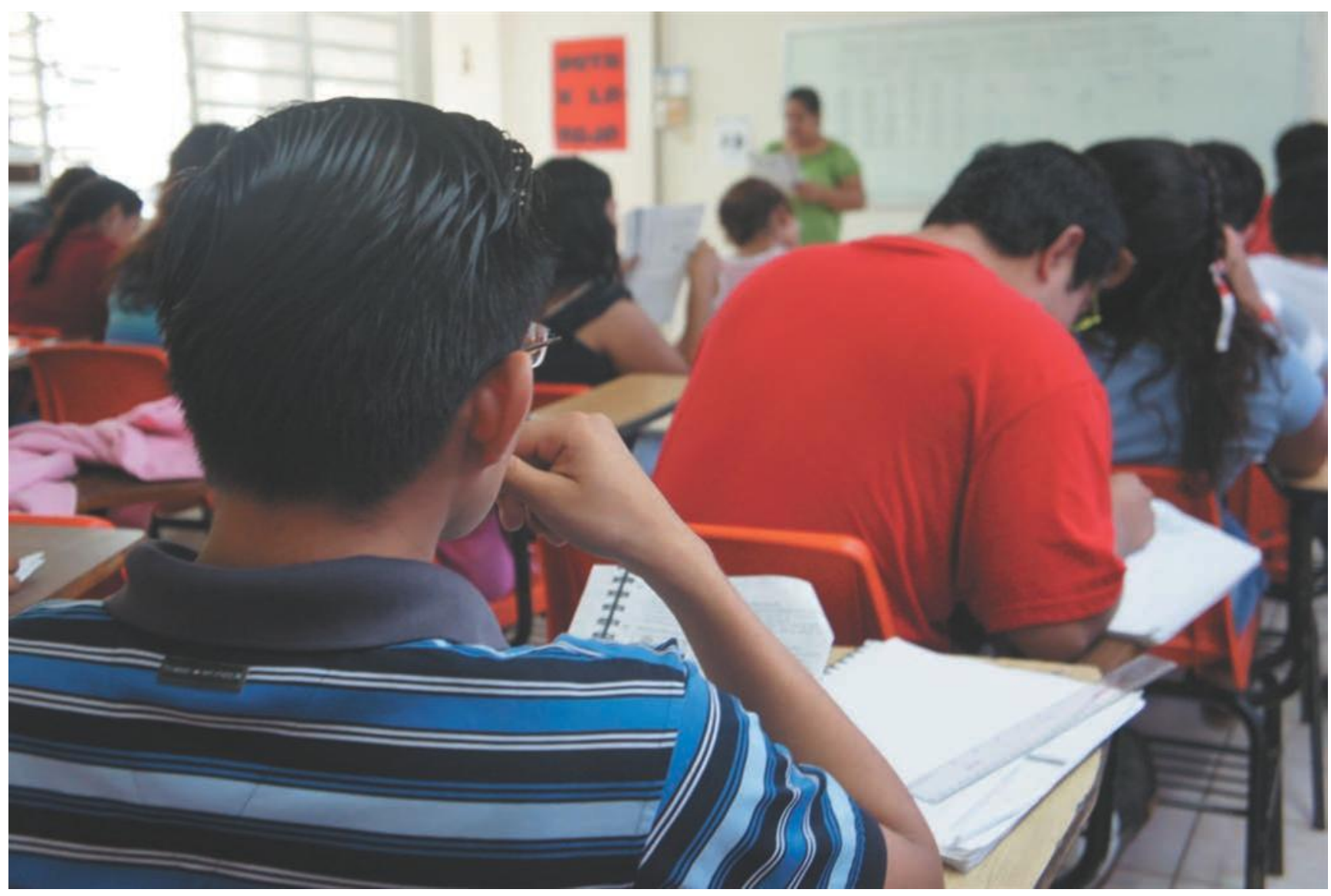

de metacognición de estudiantes de la Facultad de Contaduría Pública de la USTA en las asignaturas de Derecho Comercial II y Derecho Laboral, por cuanto los estudiantes, al comparar sobre experiencias anteriores, y con diversos docentes, perciben una gran diferencia en el autoanálisis de su proceso de aprendizaje que hasta ahora hacen y deja de ser exclusivo sólo entre los estudiantes disciplinados y se maniesta en todo el grupo, para la docente también es nuevo el aporte en la reexión de sus compromisos que quedan evidenciados y mueven a su acción, no quedan en la intención como sucede con las metodologías tradicionales en las que el docente es consciente de cambios pero difícilmente los realiza, la reexión acerca de los aciertos que el estudiantado encuentra en su propio desempeño toca distintos aspectos entre los que se encuentra la posibilidad de aanzar el desarrollo cognitivo, por cuanto se permitió el entendimiento completo del tema visto.
Otro resultado importante de la investigación logró determinar la mejora en la calidad del aprendizaje, de acuerdo a la percepción de la docente y de los estudiantes que lo han manifestado explícitamente con los instrumentos aplicados al expresar su percepción de mejora en el rendimiento del grupo incluido en la investigación, y al hacer una comparación entre las dos experiencias de la investigación, se muestran el mejoramiento promedio del último grupo en el que se agrega el uso de foros, seguimiento a la participación de los estudiantes y la aplicación de la didáctica de caso con respecto a los resultados en nota obtenidos en todas las experiencias. Así mismo, en múltiples expresiones como en las entrevistas y portafolios, el estudiante percibe diversas ventajas en el uso de la plataforma y en benecio de la experiencia acompañada de tecnología, estas opiniones se encuentran inuenciadas por diversos aspectos, entre ellos, la estructura de la asignatura a través de un protocolo, la aplica- 
ción de la didáctica de caso, el acompañamiento de la plataforma Moodle con sus ventajas enfocadas al acompañamiento de educación por medios tecnológicos, la inmersión del estudiante en la tecnología y la posibilidad de aprovechar Internet como medio de contacto, investigación y otros, el uso del portafolio virtual que reduce manejo de volúmenes de información y costos y, por último, la mejora de las relaciones grupales, tanto de intervención como de participación colaborativa, todo lo anterior reforzado por la cantidad de expresiones de ventajas en la entrevista y portafolios, supera notablemente los otros ítems 2:1 y hasta 4:1, esto inclina la opinión hacia una experiencia positiva sustentada por el nivel de los comentarios realizados por los estudiantes y que son muy signicativos como experiencia para la investigación realizada.

\section{Conclusiones}

Con respecto al uso de la tecnología, al tratar de incorporarla en el módulo de las materias de derecho que se llevan en el sistema de créditos académicos el estudiante capta la no lejanía de la docente en su tiempo independiente, por la realimentación que puede recibir en cualquier momento a través de correos enviados, y un acompañamiento de los otros compañeros con participación en herramientas como Foros, o Chat, por la expresión de algunos de ellos también la asincronía en el tiempo, les permite a un alto porcentaje de estudiantes que trabajan, la posibilidad de manejar sus tiempos de disponibilidad más adecuadamente, también motiva a que el estudiante al estar inmerso en la plataforma pueda acudir a resolver inquietudes en Internet, con búsquedas de información, traducciones, interacción con otras personas, presentación de inquietudes al docente con correos - Messenger, y varias ventajas más que crecen constantemente a través de servicios nuevos, los estudiantes expresan que la tecnología es una avance para la educación y para algunos de ellos es muy fácil de usar, la participación en el foro aportado por Moodle fue también uno de los mejores aspectos del proceso realizado, los estudiantes ingresaban a él y participaban desde la Universidad o desde lugares remotos como su casa, trabajo o café Internet dejaron atrás el obstáculo y la excusa del corto tiempo para reunirse con sus compañeros a trabajar en el tiempo independiente.

El acceso a la tecnología es el principal comentario en las desventajas expresadas por los estudiantes, esto será superado necesariamente por la compra personal y el desarrollo de centros de tecnología en las ciudades que provean préstamos de hardware y comunicaciones, así como soluciones alternas que presentará la Universidad al incremento en la demanda del servicio de computadores, otra desventaja tiene que ver con el entendimiento de la plataforma, esto será resuelto por la 16 cación de asignaturas que la usen en la Universidad, cursos de manejo de la platafor- ma, y refuerzo en las capacitaciones que se im-

partirán en el transcurso del semestre, por último es evidente que la tecnología es sensible a fallos, de conexión por ejemplo, de caídas de la página, y otras que se han presentado según los estudiantes en momentos críticos, como aquellos que han separado un tiempo especíco y no han podido llevar a cabo su participación, dicultándose su próximo acceso y demás.

Y con respecto a los tres módulos desarrollados se expresan resultados en cada uno de ellos: con respecto a la carpeta docente, componente muy importante de su uso, al describir los compromisos, la docente evidencia la necesidad de mejorar su primera experiencia dándole valor a la carpeta docente por cuanto uno de sus objetivos primordiales consiste en la reexión de su práctica docente y el mejoramiento que pueda hacer sobre sus reexiones a futuros grupos de estudiantes, este

"proceso de reexión encierra el hecho de que su elaboración permite profundizar 
en el paradigma del profesor reexivo, que basa su mejora en la praxis, es decir, en una acción contrastada con las evidencias y los datos que le permiten afrontar los cambios con una cierta seguridad y generando, al mismo tiempo, conocimiento sobre la propia enseñanza" (Schön, 1992),

También opina Schön que no podemos olvidar que la propia estrategia permite ser utilizada para acreditar la profesionalidad de los profesores universitarios, utilizándose bien como sistema de evaluación en cursos de formación relacionados exclusivamente con la docencia, bien como sistema de promoción, reconocimiento o incentivación.

Algo que la docente misma detecta es que al llevar la carpeta docente en la plataforma le permite organizar su material de clase y tener acceso a él desde cualquier lugar para enviar información a los estudiantes, para asignar tareas o trabajos y para mejorarlo y actualizarlo desde la misma plataforma.

Con respecto a los resultados del portafolio del estudiante, los estudiantes evidenciaron sucientemente en los análisis realizados a los instrumentos aplicados las bondades del mismo, y su mejora metacognitiva, asumieron, cada vez mejor, con el transcurso del núcleo construyeron para ese momento en su propio proceso de aprendizaje, por supuesto, esto es sólo un comienzo de algo que debe ser un proceso que permitiría en el tiempo para los docentes poder recibir grupos autorregulados en los que se aprovechan los tiempos presenciales de mejor manera, en la comprensión con respecto al portafolio virtual, la docente aclara que facilitó no sólo el trabajo de los estudiantes, sino su revisión, pues transportar 45 ó 50 carpetas para revisar, es una labor dispendiosa y en muchos casos imposible de realizar en cada corte, debido a que los estudiantes de manera equivocada tal vez por la multiplicidad de instrucciones por parte de los docentes, comparando con las experiencias anteriores se limitaban a resumir lo visto en clase, y condensarlo en una carpeta física.

Por último otra ventaja del portafolio virtual manifestada por los estudiantes en la plataforma y en la entrevista realizada fue la disminución de costos, porque ya no es necesaria la impresión de la información sino que puede ser consultada por el docente en la plataforma y además interactuar con el estudiante.

Con respecto al tercer módulo desarrollado y llamado de seguimiento, este se realizó por estudiante, actividad y grupo, lo que permitió observar el uso de la plataforma y motivó a los estudiantes en la interacción con la plataforma y corregir en otros su participación en las actividades realizadas, al nal, la importancia de esta herramienta radicó en la facilidad para el mismo docente en el agrupamiento de la información en unas estadísticas claras y de toma de decisiones, que fueron importantes para el mejoramiento de su asignatura.

\section{BIBLIOGRAFÍA}

Brown, A. L. (et. al). (1983). Learning, Remembering and Understanding. En P. H. Mussen. Editorial Handbook of Child Psychology. Nueva York: Wiley \& Sons. 3, 106107

Schön, D. A. (1983). El profesional reexivo. Cómo piensan los profesionales cuando actúan. Barcelona: Paidós.

Schön, D. A. (1983). The reective practitioner. Nueva York: Basis Books.

Cómo citar este artículo:

Sarmiento, J. A. Implantación de los portafolios virtuales de aprendizaje y de enseñanza en asignaturas de educación superior. Espiral, Revista de Docencia e Investigación. 2, (1), 29 - 40 
